\title{
Orbital Euclidean stability of the solutions of impulsive equations on the impulsive moments
}

\author{
Katya Georgieva Dishlieva \\ Faculty of Applied Mathematics and Informatics, Technical University of Sofia, Sofia, Bulgaria
}

Email address:

kgd@tu-sofia.bg

\section{To cite this article:}

Katya Georgieva Dishlieva. Orbital Euclidean Stability of the Solutions of Impulsive Equations on the Impulsive Moments. Pure and Applied Mathematics Journal. Vol. 4, No. 1, 2015, pp. 1-8. doi: 10.11648/j.pamj.20150401.11

\begin{abstract}
Curves given in a parametric form are studied in this paper. Curves are continuous on the left in the general case. Their corresponding parameters belong to the definitional intervals which is possible to not coincide for the different curves. Moreover, the points of discontinuity (if they exist) are first kind (jump discontinuity) and they are specific for each curve. Upper estimates of the Euclidean distance between two such curves are found. The results obtained are used in studies of the solutions of impulsive differential equations. Sufficient conditions for the orbital Euclidean stability of the solutions of such equations in respect to the impulsive effects on the initial condition and impulive moments are found. This type of stability is introduced and studied here for the first time.
\end{abstract}

Keywords: Euclidean Distance, Parametric Curves, Impulsive Differential Equations Orbital Euclidean Stability

\section{Introduction}

Finding the upper estimates of the Euclidean distance between parametric curves (including the case where the curves are piecewise continuous) is an important task. For the convenience, we will consider that the parameter of these curves represents the time. Qualitative research related to the orbital Euclidean stability of the solutions of impulsive differential equations can start after finding the above-mentioned estimates and more precise of the technology for their receiving. It is known that, the trajectories of these equations are curves which are piecewise continuous. They are continuous on the left hand side in their corresponding interval of existence. These points of discontinuity are first kind (see [2], [3], [10], [12], [14], [19] and [24]). In the case where the differential equations have variable impulsive moments their non coinciding solutions (trajectories) possess different sets of breakpoints (see [4], [5], [8], [9] and [16]). Therefore, in the paper, we explore and assesses the Euclidean distance between parametric curves which are piecewise continuous on their left hand side and which possess specific (own) moments of discontinuity of the first kind. The obtained results are applied to the study of orbital Euclidean stability which is specific for the equations with impulsive effects and is introduced in this paper. Should be noted that the impulsive differential equations are convenient mathematical apparatus for a description of dynamic phenomena subjected to the discrete short term external influences. Due to its wide application, the qualitative properties of these equations are examined seriously (see [1], [7], [11], [13], [15] - [18], [20] - [23] and [25]).

\section{Preliminary Remarks and Results}

We will use the following notations. Let the points $a\left(a_{1}, a_{2}, \ldots, a_{n}\right)$ and $b\left(b_{1}, b_{2}, \ldots, b_{n}\right) \in R^{n}$. Then their $\operatorname{dot}$ product will be denoted by:

$$
\langle a, b\rangle=a_{1} b_{1}+a_{2} b_{2}+\ldots+a_{n} b_{n} .
$$

Euclidean distance between both points is:

$$
\rho(a, b)=\sqrt{\left(a_{1}-b_{1}\right)^{2}+\left(a_{2}-b_{2}\right)^{2}+\ldots+\left(a_{n}-b_{n}\right)^{2}} .
$$

Euclidean norm $\|a\|$ of point $a$ is

$$
\|a\|=\langle a, a\rangle^{1 / 2}=\sqrt{a_{1}{ }^{2}+a_{2}{ }^{2}+\ldots+a_{n}{ }^{2}} .
$$

The next equality is valid

$$
\|a-b\|=\rho(a, b) .
$$


Let the nonempty sets $A, B \subset R^{n}$. The Euclidean distance between both sets is:

$$
\rho_{E}(A, B)=\inf \{\inf \{\rho(a, b), a \in A\}, b \in B\} .
$$

If at least one of the sets $A$ and $B$ is empty, then for the convenience, we will consider that $\rho_{E}(A, B)=0$. Further, by $\partial A$ and $\bar{A}$ are denoted the contour and closure of set $A$, respectively.

Remark 1. The next properties are valid for the Euclidean distance between the sets in $R^{n}$. Let the sets $A, B, C$, $D \subset R^{n}$ and constant $\lambda \in R$. Then:

$1.0 \leq \rho_{E}(A, B)<\infty$;

2. If $A \cap B \neq \varnothing \Rightarrow \rho_{E}(A, B)=0$;

3. $\rho_{E}(A, B)=0 \Leftrightarrow\left(\exists\left\{a_{n}\right\} \subset A, \exists\left\{b_{n}\right\} \subset B\right): \lim _{n \rightarrow \infty} \rho\left(a_{n}, b_{n}\right)=0$;

4. If $\rho_{E}(A, B)=0$ and $A, B$ are bounded $\Rightarrow \bar{A} \cap \bar{B} \neq \varnothing$;

5. If $\bar{A} \cap \bar{B} \neq \varnothing \Rightarrow \rho_{E}(A, B)=0$;

6. $\rho_{E}(\bar{A}, \bar{B})=\rho_{E}(A, B)$;

7. $\rho_{E}(A, B)=\rho_{E}(B, A)$;

8. $\rho_{E}(\lambda . A, \lambda . B)=|\lambda| . \rho_{E}(A, B)$;

9. If $\varnothing \neq A \subset B \quad$ and $\quad \varnothing \neq C \subset D$ $\Rightarrow \rho_{E}(A, C) \geq \rho_{E}(B, D)$;

10. If set $A$ is bounded and $\rho_{E}(A, B)<\infty$, then set $B$ is also bounded.

Theorem 1. Suppose that the nonempty sets $A_{1}, A_{2}, \ldots, A_{k}$, $B_{1}, B_{2}, \ldots, B_{s} \subset R^{n}$

then

$$
\begin{gathered}
\rho_{E}\left(\bigcup_{p=1, \ldots, \mathrm{k}} A_{p}, \bigcup_{q=1, \ldots, \mathrm{s}} B_{q}\right) \\
\leq \min \left\{\rho_{E}\left(A_{p}, B_{q}\right) ; p=1,2, \ldots, \mathrm{k}, q=1,2, \ldots, \mathrm{s}\right\} .
\end{gathered}
$$

Proof. Since

$$
(\forall i=1,2, \ldots, k) \Rightarrow A_{i} \subset \bigcup_{p=1, \ldots, \mathrm{k}} A_{p}
$$

and

$$
(\forall j=1,2, \ldots, \mathrm{s}) \Rightarrow B_{j} \subset \bigcup_{q=1, \ldots, \mathrm{s}} B_{q},
$$

then using property 9 of the previous remark, we obtain

$$
\begin{gathered}
\rho_{E}\left(\bigcup_{p=1, \ldots, \mathrm{k}} A_{p}, \bigcup_{q=1, \ldots, \mathrm{s}} B_{q}\right) \\
\leq \rho_{E}\left(A_{i}, B_{j}\right), i=1,2, \ldots, k, j=1,2, \ldots, s .
\end{gathered}
$$

From the inequalities above, the Theorem 1 is true.

Let the functions $g, g^{*}: R^{+} \rightarrow R^{n}$ and the constants $T_{0}, T_{1}, T_{0}^{*}, T_{1}^{*} \in R^{+}$. We introduce the parametric curves:

$$
\gamma\left[T_{0}, T_{1}\right]=\left\{\begin{array}{l}
\left\{g(t) ; T_{0} \leq t \leq T_{1}\right\}, T_{0} \leq T_{1} ; \\
\varnothing, T_{0}>T_{1}
\end{array}\right.
$$

and

$$
\gamma^{*}\left[T_{0}^{*}, T_{1}^{*}\right]=\left\{\begin{array}{l}
\left\{g^{*}(t) ; T_{0}^{*} \leq t \leq T_{1}^{*}\right\}, T_{0}^{*} \leq T_{1}^{*} \\
\varnothing, T_{0}^{*}>T_{1}^{*} .
\end{array}\right.
$$

Similarly, we introduce the curves:

$$
\begin{gathered}
\gamma\left(T_{0}, T_{1}\right], \gamma\left[T_{0}, T_{1}\right), \gamma\left(T_{0}, T_{1}\right), \\
\gamma^{*}\left(T_{0}^{*}, T_{1}^{*}\right], \gamma^{*}\left[T_{0}^{*}, T_{1}^{*}\right), \gamma^{*}\left(T_{0}^{*}, T_{1}^{*}\right),
\end{gathered}
$$

defined in the half open and open intervals, respectively.

Remark 2. Let $0 \leq T_{0} \leq T_{1}$ and $0 \leq T_{0}^{*} \leq T_{1}^{*}$. The following definitional equations, relating to the Euclidean distance between the curves $\gamma^{*}\left[T_{0}^{*}, T_{1}^{*}\right]$ and $\gamma\left[T_{0}, T_{1}\right]$, and the uniform distance between the curves $\gamma^{*}\left[T_{0}, T_{1}\right]$ and $\gamma\left[T_{0}, T_{1}\right]$, are valid respectively:

$$
\begin{gathered}
\rho_{E}\left(\gamma^{*}\left[T_{0}^{*}, T_{1}^{*}\right], \gamma\left[T_{0}, T_{1}\right]\right) \\
=\inf \left\{\inf \left\{\rho\left(g^{*}\left(t^{*}\right), g(t)\right), T_{0}^{*} \leq t^{*} \leq T_{1}^{*}\right\}, T_{0} \leq t \leq T\right\} \\
\rho_{R}\left(\gamma^{*}\left[T_{0}, T_{1}\right], \gamma\left[T_{0}, T_{1}\right]\right)=\sup \left\{\rho\left(g^{*}(t), \mathrm{g}(t)\right), T_{0} \leq t \leq T_{1}\right\} \\
=\sup \left\{\left\|g^{*}(t)-\mathrm{g}(t)\right\|, T_{0} \leq t \leq T_{1}\right\}
\end{gathered}
$$

As seen from the remark above, the uniform distance is defined only when the curves have a common definitional interval. Similar definitional equations for the Euclidean and uniform distance between the curves are valid when they are defined in the half-open and open intervals. In the next two theorems, we will use the notations:

$$
\begin{aligned}
& T_{0}^{\min }=\min \left\{T_{0}^{*}, T_{0}\right\}, T_{0}^{\max }=\max \left\{T_{0}^{*}, T_{0}\right\}, \\
& T_{1}^{\min }=\min \left\{T_{1}^{*}, T_{1}\right\}, T_{1}^{\max }=\max \left\{T_{1}^{*}, T_{1}\right\} .
\end{aligned}
$$

Theorem 2. Suppose that

1. The functions $g, g^{*} \in C\left[R^{+}, R^{n}\right]$.

2. The inequality $T_{0}^{\max } \leq T_{1}^{\min }$ is satisfied.

Then the following estimate valid

$$
\begin{gathered}
\rho_{E}\left(\gamma^{*}\left[T_{0}^{*}, T_{1}^{*}\right], \gamma\left[T_{0}, T_{1}\right]\right) \\
\leq \rho_{R}\left(\gamma^{*}\left[T_{0}^{\max }, T_{1}^{\min }\right], \gamma\left[T_{0}^{\max }, T_{1}^{\min }\right]\right) .
\end{gathered}
$$

Proof. The statement follows from Remark 1 and Remark 2. Actually we have 


$$
\begin{gathered}
\rho_{E}\left(\gamma^{*}\left[T_{0}^{*}, T_{1}^{*}\right], \gamma\left[T_{0}, T_{1}\right]\right) \\
=\rho_{E}\left(\gamma^{*}\left[T_{0}^{\min }, T_{0}^{\max }\right] \cup \gamma^{*}\left[T_{0}^{\max }, T_{1}^{\min }\right] \cup \gamma^{*}\left[T_{1}^{\min }, T_{1}^{\max }\right],\right. \\
\left.\gamma\left[T_{0}^{\min }, T_{0}^{\max }\right] \cup \gamma\left[T_{0}^{\max }, T_{1}^{\min }\right] \cup \gamma\left[T_{1}^{\min }, T_{1}^{\max }\right]\right) \\
\leq \rho_{E}\left(\gamma^{*}\left[T_{0}^{\max }, T_{1}^{\min }\right], \gamma\left[T_{0}^{\max }, T_{1}^{\min }\right]\right) \\
=\inf \left\{\inf \left\{\rho\left(g^{*}\left(t^{*}\right), g(t)\right), T_{0}^{\max } \leq t^{*} \leq T_{1}^{\min }\right\},\right. \\
\left.T_{0}^{\max } \leq t \leq T_{1}^{\min }\right\} \leq \inf \left\{\rho\left(g^{*}(t), g(t)\right), T_{0}^{\max } \leq t \leq T_{1}^{\min }\right\} \\
\leq \sup \left\{\rho\left(g^{*}(t), g(t)\right), T_{0}^{\max } \leq t \leq T_{1}^{\min }\right\} \\
=\rho_{R}\left(\gamma^{*}\left[T_{0}^{\max }, T_{1}^{\min }\right], \gamma\left[T_{0}^{\max }, T_{1}^{\min }\right]\right) .
\end{gathered}
$$

The Theorem is proved.

The next theorem enhances the previous.

Theorem 3. Suppose that:

1.The functions $g, g^{*}: R^{+} \rightarrow R^{n}$ and they are continuous on the left hand side in $R^{+}$.

2.The inequality $T_{0}^{\max } \leq T_{1}^{\min }$ is satisfied.

Then the following estimate is valid:

$$
\begin{gathered}
\rho_{E}\left(\gamma^{*}\left(T_{0}^{*}, T_{1}^{*}\right], \gamma\left(T_{0}, T_{1}\right]\right) \\
\leq \min \left\{\rho_{R}\left(\gamma^{*}\left(T_{0}^{\max }, T_{1}^{\min }\right], \gamma\left(T_{0}^{\max }, T_{1}^{\min }\right]\right),\right. \\
\rho_{E}\left(\mathrm{~g}^{*}\left(T_{0}^{*}+0\right), \gamma\left(T_{0}, T_{0}^{*}\right]\right), \rho_{E}\left(\mathrm{~g}\left(T_{0}+0\right), \gamma^{*}\left(T_{0}^{*}, T_{0}\right]\right), \\
\left.\rho_{E}\left(\mathrm{~g}\left(T_{1}\right), \gamma^{*}\left(T_{1}, T_{1}^{*}\right]\right), \rho_{E}\left(\mathrm{~g}^{*}\left(T_{1}^{*}\right), \gamma\left(T_{1}^{*}, T_{1}\right]\right)\right\} .
\end{gathered}
$$

Proof. We will prove the statement of theorem under the additional assumption that the next inequalities are valid:

$$
T_{0}^{*} \leq T_{0} \text { and } T_{1}^{*} \leq T_{1} .
$$

The other three cases are considered similarly. It is clear that in this case the intervals

$$
T_{0} \leq t<T_{0}^{*}=\varnothing \text { and } T_{1}<t \leq T_{1}^{*}=\varnothing .
$$

Therefore

$$
\rho_{E}\left(\mathrm{~g}^{*}\left(T_{0}^{*}+0\right), \gamma\left(T_{0}, T_{0}^{*}\right]\right)=\rho_{E}\left(\mathrm{~g}^{*}\left(T_{0}^{*}+0\right), \varnothing\right)=0
$$

and

$$
\rho_{E}\left(\mathrm{~g}\left(T_{1}\right), \gamma^{*}\left(T_{1}, T_{1}^{*}\right]\right)=\rho_{E}\left(\mathrm{~g}\left(T_{1}\right), \varnothing\right)=0 .
$$

Taking into account that function $g$ is continuous on the left hand side at a point $T_{0}$, we deduce that

$$
\overline{\gamma\left(T_{0}, T_{1}\right]}=g\left(T_{0}+0\right) \cup \gamma\left(T_{0}, T_{1}\right] .
$$

Analogously

$$
\overline{\gamma^{*}\left(T_{0}^{*}, T_{1}^{*}\right]}=g^{*}\left(T_{0}^{*}+0\right) \cup \gamma^{*}\left(T_{0}^{*}, T_{1}^{*}\right] .
$$

We apply property 6 of Remark 1 and we derive

$$
\begin{gathered}
\rho_{E}\left(\gamma^{*}\left(T_{0}^{*}, T_{1}^{*}\right], \gamma\left(T_{0}, T_{1}\right]\right)=\rho_{E}\left(\overline{\gamma^{*}\left(T_{0}^{*}, T_{1}^{*}\right]}, \overline{\gamma\left(T_{0}, T_{1}\right]}\right) \\
=\rho_{E}\left(g^{*}\left(T_{0}^{*}+0\right) \cup \gamma^{*}\left(T_{0}^{*}, T_{1}^{*}\right], g\left(T_{0}+0\right) \cup \gamma\left(T_{0}, T_{1}\right]\right) \\
=\rho_{E}\left(g\left(T_{0}^{*}+0\right) \cup \gamma^{*}\left(T_{0}^{*}, T_{0}\right] \cup \gamma^{*}\left(T_{0}, T_{1}^{*}\right] \cup g^{*}\left(T_{1}^{*}\right),\right. \\
\left.g\left(T_{0}+0\right) \cup \gamma\left(T_{0}, T_{1}^{*}\right] \cup \gamma\left(T_{1}^{*}, T_{1}\right] \cup g\left(T_{1}\right)\right) .
\end{gathered}
$$

Using Theorem 1 , by the equality above, we receive:

$$
\begin{gathered}
\rho_{E}\left(\gamma^{*}\left(T_{0}^{*}, T_{1}^{*}\right], \gamma\left(T_{0}, T_{1}\right]\right) \leq \min \left\{\rho_{E}\left(\gamma^{*}\left(T_{0}^{*}, T_{0}\right], g\left(T_{0}+0\right)\right),\right. \\
\left.\rho_{E}\left(\gamma^{*}\left(T_{0}, T_{1}^{*}\right], \gamma\left(T_{0}, T_{1}^{*}\right]\right), \rho_{E}\left(g^{*}\left(T_{1}^{*}\right), \gamma\left(T_{1}^{*}, T_{1}\right]\right)\right\},
\end{gathered}
$$

from where, as we get into account (1) and (2), we find:

$$
\begin{gathered}
\rho_{E}\left(\gamma^{*}\left(T_{0}^{*}, T_{1}^{*}\right], \gamma\left(T_{0}, T_{1}\right]\right) \leq \min \left\{\rho_{E}\left(\gamma^{*}\left(T_{0}^{*}, T_{0}\right], g\left(T_{0}+0\right)\right),\right. \\
\rho_{R}\left(\gamma^{*}\left(T_{0}, T_{1}^{*}\right], \gamma\left(T_{0}, T_{1}^{*}\right]\right), \rho_{E}\left(g^{*}\left(T_{1}^{*}\right), \gamma\left(T_{1}^{*}, T_{1}\right]\right), \\
\left.\rho_{E}\left(\mathrm{~g}^{*}\left(T_{0}^{*}+0\right), \gamma\left(T_{0}, T_{0}^{*}\right]\right), \rho_{E}\left(\mathrm{~g}\left(T_{1}\right), \gamma^{*}\left(T_{1}, T_{1}^{*}\right]\right)\right\} .
\end{gathered}
$$

The Theorem 3 is proved.

Similarly we prove the statement:

Theorem 4. Suppose that:

1. The functions $g, g^{*}: R^{+} \rightarrow R^{n}$ are continuous on the right hand side in $R^{+}$.

2. The inequality $T_{0}^{\max } \leq T_{1}^{\min }$ is satisfied.

Then the next estimate is valid:

$$
\begin{gathered}
\rho_{E}\left(\gamma^{*}\left[T_{0}^{*}, T_{1}^{*}\right), \gamma\left[T_{0}, T_{1}\right)\right) \\
\leq \min \left\{\rho_{R}\left(\gamma^{*}\left[T_{0}^{\max }, T_{1}^{\min }\right), \gamma\left[T_{0}^{\max }, T_{1}^{\min }\right)\right),\right. \\
\rho_{E}\left(\mathrm{~g}^{*}\left(T_{0}^{*}\right), \gamma\left[T_{0}, T_{0}^{*}\right)\right), \rho_{E}\left(\mathrm{~g}\left(T_{0}\right), \gamma^{*}\left[T_{0}^{*}, T_{0}\right)\right), \\
\left.\rho_{E}\left(\mathrm{~g}\left(T_{1}-0\right), \gamma^{*}\left[T_{1}, T_{1}^{*}\right)\right), \rho_{E}\left(\mathrm{~g}^{*}\left(T_{1}^{*}-0\right), \gamma\left[T_{1}^{*}, T_{1}\right)\right)\right\} .
\end{gathered}
$$

As a consequence of the theorem above, we will formulate the following statement relating to the Euclidean distance between continuous curves.

Theorem 5. Suppose that:

1. The functions $g, g^{*} \in C\left[R^{+}, R^{n}\right]$.

2. The inequality $T_{0}^{\max } \leq T_{1}^{\min }$ is satisfied.

Then the next estimate is valid:

$$
\begin{aligned}
& \rho_{E}\left(\gamma^{*}\left[T_{0}^{*}, T_{1}^{*}\right], \gamma\left[T_{0}, T_{1}\right]\right)=\rho_{E}\left(\gamma^{*}\left(T_{0}^{*}, T_{1}^{*}\right], \gamma\left(T_{0}, T\right]\right) \\
= & \rho_{E}\left(\gamma^{*}\left[T_{0}^{*}, T_{1}^{*}\right), \gamma\left[T_{0}, T\right)\right)=\rho_{E}\left(\gamma^{*}\left(T_{0}^{*}, T_{1}^{*}\right), \gamma\left(T_{0}, T\right)\right)
\end{aligned}
$$




$$
\begin{gathered}
\leq \min \left\{\rho_{R}\left(\gamma^{*}\left[T_{0}^{\max }, T_{1}^{\min }\right], \gamma\left[T_{0}^{\max }, T_{1}^{\min }\right]\right),\right. \\
\rho_{E}\left(\mathrm{~g}\left(T_{0}\right), \gamma^{*}\left[T_{0}^{*}, T_{0}\right]\right), \rho_{E}\left(\mathrm{~g}^{*}\left(T_{0}^{*}\right), \gamma\left[T_{0}, T_{0}^{*}\right]\right), \\
\left.\rho_{E}\left(\mathrm{~g}\left(T_{1}\right), \gamma^{*}\left[T_{1}, T_{1}^{*}\right]\right), \quad \rho_{E}\left(\mathrm{~g}^{*}\left(T_{1}^{*}\right), \gamma\left[T_{1}^{*}, T_{1}\right]\right)\right\} .
\end{gathered}
$$

The following theorem summarizes Theorem 3 and Theorem 4.

Theorem 6. Suppose that:

1. The functions $g, g^{*}: R^{+} \rightarrow R^{n}$.

2. There exists a number $k \in N$, such that the following inequalities are satisfied:

$$
\begin{gathered}
0<T_{0}^{*}<T_{1}^{*}<\ldots<T_{k}^{*} ; \\
0<T_{0}<T_{1}<\ldots<T_{k} ; \\
0<T_{0}^{\max }<T_{1}^{\min } \leq T_{1}^{\max }<T_{2}^{\min } \leq T_{2}^{\max }<\ldots<T_{k}^{\min } \leq T_{k}^{\max },
\end{gathered}
$$

where

$$
\begin{gathered}
T_{0}^{\min }=\min \left\{T_{0}^{*}, \mathrm{~T}_{0}\right\}, \quad T_{0}^{\max }=\max \left\{T_{0}^{*}, \mathrm{~T}_{0}\right\}, \ldots, \\
T_{k}^{\min }=\min \left\{T_{k}^{*}, \mathrm{~T}_{k}\right\}, T_{k}^{\max }=\max \left\{T_{k}^{*}, \mathrm{~T}_{k}\right\} .
\end{gathered}
$$

Then:

1. If the functions $g$ and $g^{*}$ are continuous on the left hand side in $R^{+}$, then the next inequality is valid:

$$
\begin{gathered}
\rho_{E}\left(\gamma^{*}\left(T_{0}^{*}, T_{k}^{*}\right], \gamma\left(T_{0}, T_{k}\right]\right) \\
\leq \min \left\{\rho_{R}\left(\gamma^{*}\left(T_{i-1}^{\max }, T_{i}^{\min }\right], \gamma\left(T_{i-1}^{\max }, T_{i}^{\min }\right]\right),\right. \\
\rho_{E}\left(\mathrm{~g}\left(T_{i-1}+0\right), \gamma^{*}\left(T_{i-1}^{*}, T_{i-1}\right]\right), \rho_{E}\left(\mathrm{~g}^{*}\left(T_{i-1}^{*}+0\right), \gamma\left(T_{i-1}, T_{i-1}^{*}\right]\right), \\
\rho_{E}\left(\mathrm{~g}\left(T_{i}\right), \gamma^{*}\left(T_{i}, T_{i}^{*}\right]\right), \\
\left.\rho_{E}\left(\mathrm{~g}^{*}\left(T_{i}^{*}\right), \gamma\left(T_{i}^{*}, T_{i}\right]\right), i=1,2, \ldots, k\right\} .
\end{gathered}
$$

2. If the functions $g$ and $g^{*}$ are continuous on the right hand side in $R^{+}$, then the next inequality is valid:

$$
\begin{gathered}
\rho_{E}\left(\gamma^{*}\left[T_{0}^{*}, T_{k}^{*}\right), \gamma\left[T_{0}, T_{k}\right)\right) \\
\leq \min \left\{\rho_{R}\left(\gamma^{*}\left[T_{i-1}^{\max }, T_{i}^{\min }\right), \gamma\left[T_{i-1}^{\max }, T_{i}^{\min }\right)\right),\right. \\
\rho_{E}\left(\mathrm{~g}\left(T_{i-1}\right), \gamma^{*}\left[T_{i-1}^{*}, T_{i-1}\right)\right), \rho_{E}\left(\mathrm{~g}^{*}\left(T_{i-1}^{*}\right), \gamma\left[T_{i-1}, T_{i-1}^{*}\right)\right), \\
\rho_{E}\left(\mathrm{~g}\left(T_{i}-0\right), \gamma^{*}\left[T_{i}, T_{i}^{*}\right)\right), \\
\left.\rho_{E}\left(\mathrm{~g}^{*}\left(T_{i}^{*}-0\right), \gamma\left[T_{i}^{*}, T_{i}\right)\right), i=1,2, \ldots, k\right\} .
\end{gathered}
$$

Definiton 1.If

1. The functions $g, g^{*}: R^{+} \rightarrow R^{n}$.
2. The function $G\left(t ; T_{0}^{*}, T_{0}\right)=\rho_{E}\left(\gamma^{*}\left[T_{0}^{*}, t\right], \gamma\left[T_{0}, t\right]\right)$, where $t, T_{0}^{*}, T_{0} \in R^{+}$.

We will say that the functions $g$ and $g^{*}$ are:

1. Euclidean equivalent if

$$
\left(\exists T_{0}^{*}, T_{0} \in R^{+}\right): \lim _{t \rightarrow \infty} G\left(t ; T_{0}^{*}, T_{0}\right)=0 .
$$

In this case, we denote $g^{*} \square g$.

2. Uniformly Euclidean equivalent if

$$
\begin{gathered}
\left(\exists \Delta_{0}^{*}, \Delta_{0} \in R^{+}\right):\left(\forall T_{0}^{*} \geq \Delta_{0}^{*}, \forall T_{0} \geq \Delta_{0}\right) \\
\Rightarrow \lim _{t \rightarrow \infty} G\left(t ; T_{0}^{*}, T_{0}\right)=0 .
\end{gathered}
$$

The notation in this case is $g^{*} \approx g$.

Remark 3. Let $\max \left\{T_{0}^{*}, T_{0}\right\}=T_{0}^{\max } \leq t_{1}<t_{2}$. Then

$$
\begin{gathered}
G\left(t_{2} ; T_{0}^{*}, T_{0}\right)=\rho_{E}\left(\gamma^{*}\left[T_{0}^{*}, t_{2}\right], \gamma\left[T_{0}, t_{2}\right]\right) \\
=\rho_{E}\left(\gamma^{*}\left[T_{0}^{*}, t_{1}\right] \cup \gamma^{*}\left[t_{1}, t_{2}\right], \gamma\left[T_{0}, t_{1}\right] \cup \gamma\left[t_{1}, t_{2}\right]\right) \\
\leq \rho_{E}\left(\gamma^{*}\left[T_{0}^{*}, t_{1}\right], \gamma\left[T_{0}, t_{1}\right]\right)=G\left(t_{1} ; T_{0}^{*}, T_{0}\right),
\end{gathered}
$$

i.e. function $G$ is monotonically decreasing for $t \geq T_{0}^{\max }$. Furthermore, $G\left(t_{1} ; T_{0}^{*}, T_{0}\right) \geq 0$. Consequently, the limit $\lim _{t \rightarrow \infty} G\left(t ; T_{0}^{*}, T_{0}\right)$ always exists.

Remark 4. It is clear that if two functions are uniformly Euclidean equivalent, then they are Euclidean equivalent. The opposite is not true. Indeed, if there exists a constant $T_{1}>\max \left\{T_{0}^{*}, T_{0}\right\}$ such that:

$$
\lim _{t \rightarrow T_{1}-0} G\left(t ; T_{0}^{*}, T_{0}\right)=0 \text { and } \lim _{t \rightarrow \infty} G\left(t ; T_{1}, T_{1}\right)=\text { const }>0,
$$

then:

1. From the first equality, it follows that

$$
\lim _{t \rightarrow \infty} G\left(t ; T_{0}^{*}, T_{0}\right)=0
$$

i.e. the functions are Euclidean equivalent;

2. By the second inequality, it follows that

$$
\begin{gathered}
\left(\forall T_{01}^{*} \geq T_{1}, \forall T_{01} \geq T_{1}\right) \\
\Rightarrow \lim _{t \rightarrow \infty} G\left(t ; T_{01}^{*}, T_{01}\right) \geq \lim _{t \rightarrow \infty} G\left(t ; T_{1}, T_{1}\right)=\text { const }>0,
\end{gathered}
$$

i.e. the functions are not uniformly Euclidean equivalent.

3. Main Results

The following initial value problem of impulsive differential equations is an object of investigation in the paper:

$$
\frac{d x}{d t}=f(t, x), t_{i-1}<t \leq t_{i}
$$

$$
x\left(t_{i}+0\right)=x\left(t_{i}\right)+I_{i}\left(x\left(t_{i}\right)\right), i=1,2, \ldots
$$




$$
x\left(t_{0}\right)=x_{0},
$$

where:

- Function $f: R^{+} \times D \rightarrow R^{n}$;

- $D$ is a nonempty domain in $R^{n}$;

- The impulsive functions $I_{i}: D \rightarrow R^{n},\left(I d+I_{i}\right): D \rightarrow D$, $i=1,2, \ldots$;

- $I d$ is an identity in $R^{n}$;

- An initial point $\left(t_{0}, x_{0}\right) \in R^{+} \times D$;

- The moments $t_{1}, t_{2}, \ldots, t_{0}<t_{1}<t_{2}<\ldots$, are named impulsive.

The solution $x\left(t ; t_{0}, x_{0}\right)$ of problem (3), (4), (5), we define as follows:

1.1. For $t_{0} \leq t \leq t_{1}$, the solution of the considered problem coincides with the solution of problem without impulses

$$
\frac{d x}{d t}=f(t, x), \quad x\left(t_{0}\right)=x_{0} .
$$

We introduce the notation $x_{1}=x\left(t_{1} ; t_{0}, x_{0}\right)$.

1.2. At the moment $t_{1}$, the impulsive effects satisfying the equality below takes place:

$$
\begin{aligned}
x\left(t_{1}+0 ; t_{0}, x_{0}\right) & =x\left(t_{1} ; t_{0}, x_{0}\right)+I_{1}\left(x\left(t_{1} ; t_{0}, x_{0}\right)\right) \\
= & x_{1}+I_{1}\left(x_{1}\right)=x_{1}^{+}
\end{aligned}
$$

2.1. For $t_{1}<t \leq t_{2}$, the solution of problem (3), (4), (5) coincides with the solution of problem without impulses

$$
\frac{d x}{d t}=f(t, x), \quad x\left(t_{1}\right)=x_{1}^{+} .
$$

We denote by $x_{2}=x\left(t_{2} ; t_{0}, x_{0}\right)$.

2.2. At the moment $t_{2}$, the impulsive effects is performed:

$$
\begin{aligned}
x\left(t_{2}+0 ; t_{0}, x_{0}\right) & =x\left(t_{2} ; t_{0}, x_{0}\right)+I_{2}\left(x\left(t_{2} ; t_{0}, x_{0}\right)\right) \\
& =x_{2}+I_{1}\left(x_{2}\right)=x_{2}^{+}
\end{aligned}
$$

etc.

It is clear that the solution is a piecewise continuous function with breakpoints $t_{1}, t_{2}, \ldots$, in which the solution is continuous on the left hand side. Further, we will use the notations:

$$
\begin{gathered}
x_{i}=x\left(t_{i} ; t_{0}, x_{0}\right) \\
x_{i}^{+}=x\left(t_{i} ; t_{0}, x_{0}\right)+I_{i}\left(x\left(t_{i} ; t_{0}, x_{0}\right)\right)=\left(I d+I_{i}\right)\left(x_{i}\right), i=1,2, \ldots .
\end{gathered}
$$

Along with the main problem, we consider also the perturbed problem:

$$
\frac{d x}{d t}=f(t, x), t_{i-1}^{*}<t \leq t_{i}^{*}
$$

$$
x\left(t_{i}^{*}+0\right)=x\left(t_{i}^{*}\right)+I_{i}\left(x\left(t_{i}^{*}\right)\right), i=1,2, \ldots
$$

$$
x\left(t_{0}^{*}\right)=x_{0}^{*},
$$

where the initial point is $\left(t_{0}^{*}, x_{0}^{*}\right) \in R^{+} \times D$ and the impulsive moments are $t_{1}^{*}, t_{2}^{*}, \ldots, t_{0}^{*}<t_{1}^{*}<t_{2}^{*}<\ldots$. The solution of the perturbed problem is denoted by $x^{*}\left(t ; t_{0}^{*}, x_{0}^{*}\right)$.

Let the constants $T_{1}, T_{2}, T_{1}^{*}, T_{2}^{*} \in R^{+}$and $T_{1}<T_{2}, T_{1}^{*}<T_{2}^{*}$. By $\chi\left(T_{1}, T_{2}\right]$ is denoted the trajectory of problem (3), (4), (5), defined for $T_{1}<t \leq T_{2}$. Analogously, by $\chi^{*}\left(T_{1}^{*}, T_{2}^{*}\right]$ is denoted the trajectory of problem (6), (7), (8), locked in $T_{1}^{*}<t \leq T_{2}^{*}$. The following equalities are valid:

$$
\chi\left(T_{1}, T_{2}\right]=\left\{\begin{array}{l}
\left\{x\left(t ; t_{0}, x_{0}\right) ; T_{1}<t \leq T_{2}\right\}, T_{1}<T_{2} ; \\
\varnothing, T_{1} \geq T_{2}
\end{array}\right.
$$

and

$$
\chi^{*}\left(T_{1}^{*}, T_{2}^{*}\right]=\left\{\begin{array}{l}
\left\{x^{*}\left(t ; t_{0}^{*}, x_{0}^{*}\right) ; T_{1}^{*}<t \leq T_{2}^{*}\right\}, T_{1}^{*}<T_{2}^{*} \\
\varnothing, T_{1}^{*} \geq T_{2}^{*} .
\end{array}\right.
$$

We denote

$$
t_{i}^{\min }=\min \left\{t_{i}^{*}, t_{i}\right\} \text { and } t_{i}^{\max }=\max \left\{t_{i}^{*}, t_{i}\right\}, i=0,1,2, \ldots
$$

Definiton 2. We will say that the solution of the main problem (3), (4), (5) is (uniformly) orbital Euclidean stable on the initial point $\left(t_{0}, x_{0}\right)$ and the impulsive moments $t_{1}, t_{2}, \ldots$, if:

$$
\begin{gathered}
\left(\exists \delta_{t 0}, \delta_{t 1}, \ldots \in R^{+}, \delta_{t 0}+\delta_{t 1}+\ldots<\infty\right)\left(\exists \delta_{x}=\text { const } \in R^{+}\right): \\
\left(\forall\left(t_{0}^{*}, x_{0}^{*}\right) \in R^{+} \times D,\left|t_{0}^{*}-t_{0}\right|<\delta_{t 0},\left\|x_{0}^{*}-x_{0}\right\|<\delta_{x}\right) \\
\left(\forall t_{1}^{*}, t_{2}^{*}, \ldots,\left|t_{i}^{*}-t_{i}\right|<\delta_{t i}, i=1,2, \ldots\right) \Rightarrow \\
x^{*}\left(t ; t_{0}^{*}, x_{0}^{*}\right) \approx x\left(t ; t_{0}, x_{0}\right),
\end{gathered}
$$

i.e. the solutions $x\left(t ; t_{0}, x_{0}\right)$ and $x^{*}\left(t ; t_{0}^{*}, x_{0}^{*}\right)$ are (uniformly) Euclidean equivalents.

The main purpose of this study is to find the sufficient conditions which guarantee the property uniformly orbital Euclidean stability of the solutions of the considered equations. Further we will refer to the problem

$$
\frac{d x}{d t}=f(t, x), \quad x\left(t_{0}\right)=x_{0} .
$$

The solution of this problem is denoted by $\chi\left(t ; t_{0}, x_{0}\right)$. Definiton 3 . We will say that the solutions of system (9) are 
uniformly Lipschitz stable with a positive Lipschitz constant $C_{L}$, if

$$
\begin{gathered}
\left(\exists \delta_{L}=\text { const }>0\right): \\
\left(\forall t_{0} \in R^{+}\right)\left(\forall x_{0}^{\prime}, x_{0}^{\prime \prime} \in D,\left\|x_{0}^{\prime}-x_{0}^{\prime \prime}\right\|<\delta_{L}\right) \\
\Rightarrow\left\|\chi\left(t ; t_{0}, x_{0}^{\prime}\right)-\chi\left(t ; t_{0}, x_{0}^{\prime \prime}\right)\right\|<C_{L}\left\|x_{0}^{\prime}-x_{0}^{\prime \prime}\right\|, t \geq t_{0} .
\end{gathered}
$$

The uniform Lipschitz stability was introduced in 1986 by F. Dannan and S. Elaydi in [6].

We will use the following conditions:

H1.For every point $\left(t_{0}, x_{0}\right) \in R^{+} \times D$, the solution of problem (9) exists and is unique for $t \geq t_{0}$. The solution is Lipschitz stable with Lipschitz constant $C_{L}$.

H2.There exists a positive constant $C_{f}$ such that

$$
\left(\forall(t, x) \in R^{+} \times D\right) \Rightarrow\|f(t, x)\| \leq C_{f} .
$$

H3. There exists a positive constant $C_{t}$ such that $t_{i}-t_{i-1}$ $\geq C_{t}, \quad i=1,2, \ldots$.

H4.The impulsive functions $I_{1}, I_{2}, \ldots$ are equal Lipschitz, i.e. there exists a positive constant $C_{I}$ such that

$$
\left(\forall x^{\prime}, x^{\prime \prime} \in D\right) \Rightarrow\left\|I_{i}\left(x^{\prime}\right)-I_{i}\left(x^{\prime \prime}\right)\right\| \leq C_{I}\left\|x^{\prime}-x^{\prime \prime}\right\|, i=1,2, \ldots .
$$

Theorem 7. Let the conditions H1-H4 be valid.

If $C_{L} C_{I}<1$, then the solution of problem (3), (4), (5) is uniformly orbital Euclidean stable.

Proof. From condition H3, it follows that $\lim _{i \rightarrow \infty} t_{i}=\infty$ is fulfilled. We assume that:

$$
\left|t_{i}^{*}-t_{i}\right|<\delta_{t i}
$$

where $0<\delta_{t i}<C_{t}, \quad i=0,1,2, \ldots, \quad \delta_{t 0}+\delta_{t 1}+\ldots<\infty$ and

$$
\left\|x_{0}^{*}-x_{0}\right\|<\delta_{x 0}
$$

where $\delta_{x 0}>0$.

Using condition $\mathrm{H} 3$ and inequality (10) it follows

$$
t_{i-1}^{\max }<t_{i}^{\min }, i=1,2, \ldots .
$$

For convenience, we divide the proof into several parts.

Part 1.We will evaluate the norm of difference $x^{*}\left(t_{0}^{\max } ; t_{0}^{*}, x_{0}^{*}\right)-x\left(t_{0}^{\max } ; t_{0}, x_{0}\right)$. For this purpose, we will suppose that $t_{0} \leq t_{0}^{*}=t_{0}^{\max }$. The other case is considered similarly. Given all of (10), (11) and condition H2, we get

$$
\begin{aligned}
& \left\|x^{*}\left(t_{0}^{\max } ; t_{0}^{*}, x_{0}^{*}\right)-x\left(t_{0}^{\max } ; t_{0}, x_{0}\right)\right\| \\
& \leq\left\|x^{*}\left(t_{0}^{\max } ; t_{0}^{*}, x_{0}^{*}\right)-x\left(t_{0} ; t_{0}, x_{0}\right)\right\|
\end{aligned}
$$

$$
\begin{gathered}
+\left\|x\left(t_{0}^{\max } ; t_{0}, x_{0}\right)-x\left(t_{0} ; t_{0}, x_{0}\right)\right\| \\
\leq\left\|x_{0}^{*}-x_{0}\right\|+\int_{t_{0}}^{t_{t^{*}}^{*}}\left\|f\left(\tau, x\left(\tau ; t_{0}, x_{0}\right)\right) d \tau\right\| \\
\leq \delta_{x 0}+C_{f}\left|t_{0}^{*}-t_{0}\right| \leq \delta_{x 0}+C_{f} \delta_{t 0} .
\end{gathered}
$$

Part 2. We will estimate the norm of difference $x^{*}\left(t_{1}^{\mathrm{min}} ; t_{0}^{*}, x_{0}^{*}\right)-x\left(t_{1}^{\mathrm{min}} ; t_{0}, x_{0}\right)$. Without limitation, we can assume that, $\delta_{x 0}+C_{f} \delta_{t 0}<\delta_{L}$, where the constant $\delta_{L}$ satisfies Definition 3. Then from Part 1 we get

$$
\left\|x^{*}\left(t_{0}^{\max } ; t_{0}^{*}, x_{0}^{*}\right)-x\left(t_{0}^{\max } ; t_{0}, x_{0}\right)\right\|<\delta_{L} .
$$

Finally, from condition $\mathrm{H} 1$ we have

$$
\begin{gathered}
\left\|x^{*}\left(t_{1}^{\min } ; t_{0}^{*}, x_{0}^{*}\right)-x\left(t_{1}^{\min } ; t_{0}, x_{0}\right)\right\| \\
\leq\left\|x^{*}\left(t_{1}^{\min } ; t_{0}^{\max }, x^{*}\left(t_{0}^{\max } ; t_{0}^{*}, x_{0}^{*}\right)\right)-x\left(t_{1}^{\min } ; t_{0}^{\max }, x\left(t_{0}^{\max } ; t_{0}, x_{0}\right)\right)\right\| \\
\leq C_{L}\left\|x^{*}\left(t_{0}^{\max } ; t_{0}^{*}, x_{0}^{*}\right)-x\left(t_{0}^{\max } ; t_{0}, x_{0}\right)\right\| \leq C_{L} \delta_{x 0}+C_{L} C_{f} \delta_{t 0} .
\end{gathered}
$$

Part 3.We will evaluate the difference $x^{*}\left(t_{1}^{*} ; t_{0}^{*}, x_{0}^{*}\right)$ $-x\left(t_{1} ; t_{0}, x_{0}\right)$. Assume that the inequality $t_{1}^{\min }=t_{1}^{*} \leq t_{1}=t_{1}^{\max }$ is valid. Then

$$
\begin{gathered}
\left\|x^{*}\left(t_{1}^{*} ; t_{0}^{*}, x_{0}^{*}\right)-x\left(t_{1} ; t_{0}, x_{0}\right)\right\| \\
\leq\left\|x^{*}\left(t_{1}^{*} ; t_{0}^{*}, x_{0}^{*}\right)-x\left(t_{1}^{*} ; t_{0}, x_{0}\right)\right\|+\left\|x\left(t_{1}^{*} ; t_{0}, x_{0}\right)-x\left(t_{1} ; t_{0}, x_{0}\right)\right\| \\
\leq C_{L} \delta_{x 0}+C_{f}\left(C_{L}+1\right) \delta_{t 0} .
\end{gathered}
$$

Part 4.We will estimate the norm of difference

$$
x_{1}^{*+}-x_{1}^{+}=x^{*}\left(t_{1}^{*}+0 ; t_{0}^{*}, x_{0}^{*}\right)-x\left(t_{1}+0 ; t_{0}, x_{0}\right) .
$$

Let as in the previous part of the proof, we assume that $t_{1}^{*} \leq t_{1}=t_{1}^{\max }$. The consideration in the other case are similar. We consistently find

$$
\begin{aligned}
\left\|x_{1}^{*+}-x_{1}^{+}\right\| & =\left\|x^{*}\left(t_{1}^{*}+0 ; t_{0}^{*}, x_{0}^{*}\right)-x\left(t_{1}+0 ; t_{0}, x_{0}\right)\right\| \\
& =\| x^{*}\left(t_{1}^{*} ; t_{0}^{*}, x_{0}^{*}\right)+I_{1}\left(x^{*}\left(t_{1}^{*} ; t_{0}^{*}, x_{0}^{*}\right)\right)
\end{aligned}
$$

$$
\begin{gathered}
-x\left(t_{1} ; t_{0}, x_{0}\right)-I_{1}\left(x\left(t_{1} ; t_{0}, x_{0}\right)\right)\|\leq\| x^{*}\left(t_{1}^{*} ; t_{0}^{*}, x_{0}^{*}\right)-x\left(t_{1} ; t_{0}, x_{0}\right) \| \\
+\left\|I_{1}\left(x^{*}\left(t_{1}^{*} ; t_{0}^{*}, x_{0}^{*}\right)\right)-I_{1}\left(x\left(t_{1} ; t_{0}, x_{0}\right)\right)\right\| \\
\leq C_{I} C_{L} \delta_{x 0}+C_{I} C_{L} \frac{C_{f}\left(C_{L}+1\right)}{C_{L}} \delta_{t 0}=q \delta_{x 0}+q \Delta \delta_{t 0}
\end{gathered}
$$

where $q=C_{I} C_{L}$ and $\Delta=\frac{C_{f}\left(C_{L}+1\right)}{C_{L}}$. 
Part 5.Let $\delta_{x 1}=q \delta_{x 0}+q \Delta \delta_{t 0}$. By repeating the reasoning of the previous four parts of the proof we reach the estimate

$$
\left\|x_{2}^{*+}-x_{2}^{+}\right\| \leq q \delta_{x 1}+q \Delta \delta_{t 1}=q^{2} \delta_{x 0}+\Delta\left(q^{2} \delta_{t 0}+q \delta_{t 1}\right) .
$$

Similarly, for each number $k$, we obtain

$$
\left\|x_{k}^{*+}-x_{k}^{+}\right\| \leq q^{k} \delta_{x 0}+\Delta\left(q^{k} \delta_{t 0}+q^{k-1} \delta_{t 1}+\ldots+q \delta_{t(k-1)}\right), k=1,2, \ldots .
$$

Part 6.For each $t_{0}^{*}, t_{0} \in R^{+}$, we have

$$
\begin{gathered}
\lim _{t \rightarrow \infty} G\left(t, t_{0}^{*}, t_{0}\right)=\lim _{t \rightarrow \infty} \rho_{E}\left(\chi^{*}\left[t_{0}^{*}, t\right], \chi\left[t_{0}, t\right]\right) \leq \lim _{k \rightarrow \infty}\left\|x_{k}^{*+}-x_{k}^{+}\right\| \\
\leq \delta_{x 0} \cdot \lim _{k \rightarrow \infty} q^{k}+\Delta \cdot \lim _{k \rightarrow \infty}\left(q^{k} \delta_{t 0}+q^{k-1} \delta_{t 1}+\ldots+q \delta_{t(k-1)}\right) \\
=\Delta \cdot \lim _{k \rightarrow \infty}\left[\left(q^{k} \delta_{t 0}+q^{k-1} \delta_{t 1}+\ldots+q^{s} \delta_{t(k-s)}\right)\right. \\
\left.+\left(q^{s-1} \delta_{t(k-s+1)}+q^{s-2} \delta_{t(k-s+2)}+\ldots+q \delta_{t(k-1)}\right)\right] \\
\leq \Delta \cdot \lim _{k \rightarrow \infty}\left[q^{s}\left(1+q+\ldots+q^{k-s}\right)\left(\delta_{t 0}+\delta_{t 1}+\ldots+\delta_{t(k-s)}\right)\right. \\
\quad+\left(q^{s-1}+q^{s-2}+\ldots+q\right) \times \\
\left.\times \max \left\{\delta_{t i} ; i=(k-s+1),(k-s+2), \ldots,(k-1)\right\}\right] \\
\leq \Delta \cdot \frac{q^{s}}{1-q} \lim _{k \rightarrow \infty}\left(\delta_{t 0}+\delta_{t 1}+\ldots+\delta_{t(k-s)}\right) \\
\left.+\frac{\Delta}{1-q} \lim _{k \rightarrow \infty} \max \left\{\delta_{t i} ; i=(k-s+1),(k-s+2), \ldots,(k-1)\right\}\right] \\
\leq q^{s} \frac{\Delta \delta_{t}}{1-q}+\frac{\Delta}{1-q} \sup \left\{\delta_{t i} ; i=(k-s+1),(k-s+2), \ldots\right\} .(12) \\
\end{gathered}
$$

Let $\varepsilon$ be an arbitrary positive constant. Since the constant $q$ satisfies the inequalities $0<q<1$, then it is clear that

$$
\left(\exists s_{1}=s_{1}(\varepsilon) \in N\right):\left(\forall s \geq s_{1}\right) \Rightarrow q^{s} \frac{\Delta \delta_{t}}{1-q}<\frac{\varepsilon}{2} .
$$

We fix $s=s_{1}$. Since the series $\delta_{t 1}+\delta_{t 2}+\ldots$ is convergent, then it is fulfilled

$$
\begin{gathered}
\left(\exists k_{1} \in N, k_{1}>s_{1}\right): \\
\left(\forall i>k_{1}-s_{1}\right) \Rightarrow \frac{\Delta}{1-q} k_{t i}<\frac{\varepsilon}{2} \\
\Leftrightarrow \frac{\Delta}{1-q} \sup \left\{\delta_{t i} ; i=\left(k_{1}-s_{1}+1\right),\left(k_{1}-s_{1}+2\right), \ldots\right\}<\frac{\varepsilon}{2} .
\end{gathered}
$$

By (12), (13) and (14), it follows that for every $t_{0}^{*}, t_{0} \in R^{+}$, it is satisfied

$$
\lim _{t \rightarrow \infty} G\left(t, t_{0}^{*}, t_{0}\right)<\varepsilon \Leftrightarrow \lim _{t \rightarrow \infty} G\left(t, t_{0}^{*}, t_{0}\right)=0 .
$$

It means that the solution of problem (3), (4), (5) is uniformly orbital Euclidean stable.

The Theorem 7 is proved.

\section{References}

[1] Akhmet M., Beklioglu M., Ergenc T., Tkachenko V., An impulsive ratio-dependent predator-prey system with diffusion, Nonlinear Analysis: Real World Applications, Vol. 7, № 5, (2006), 1255-1267.

[2] Alzabut J., Abdeljawad T., Exponential boundedness for solutions of linear impulsive differential equations with distributed delay, International J. of Pure and Applied Mathematics, Vol. 34, № 2, (2007), 201-215.

[3] Alzabut J., Existence of periodic solutions of a type of nonlinear impulsive delay differential equations with a small parameter, J. of Nonlinear Mathematical Physics, Vol. 15, (2008), 13-21.

[4] Bainov D., Dishliev A., Population dynamics control in regard to minimizing the time necessary for the regeneration of a biomass taken away from the population, Comtes Rendus de l'Academie Bulgare Sciences, Vol. 42, № 12, (1989), 29- 32.

[5] Bainov D., Dishliev A., The phenomenon "beating" of the solutions of impulsive functional differential equations, Communications in Applied Analysis, Vol. 1, № 4, (1997), 435-441.

[6] Dannan F., Elaydi S., Lipchitz stability of nonlinear systems of differential equations, $\mathrm{J}$ of Mathematical Analysis and Applications, Vol. 113, (1986), 562-577.

[7] Darling R. W. R., Norris J. R., Differential equation approximations for Markov chains, Probability Surveys, Vol. 5, (2008), 37-79.

[8] Dishliev A., Bainov D., Continuous dependence of the solution of a system of differential equations with impulses on the impulse hypersurfaces, J. of Math. Analysis and Applications, Vol. 135, № 2, (1988), 369-382.

[9] Dishlieva K., Continuous dependence of the solutions of impulsive differential equations on the initial conditions and barrier curves, Acta Mathematica Scientia, Vol. 32, № 3, (2012), 1035-1052.

[10] Dishlieva K., Differentiability of solutions of impulsive differential equations with respect to the impulsive perturbations, Nonlinear Analysis Series B: Real World Applications, Vol. 12, № 6, (2011), 3541-3551.

[11] Dong L., Chen L., Shi P., Periodic solutions for a two-species nonautonomous competition system with diffusion and impulses, Chaos Solitons \& Fractals, Vol. 32, № 5, (2007), 1916-1926.

[12] Henderson J., Thompson H., Smoothness of solutions for boundary value problems with impulse effects, II, Math and Computer Modeling, Vol. 223, № 10, (1996), 61-69.

[13] Liu L., Ye Y., Existence and uniqueness of periodic solutions for a discrete-time SIP epidemic model with time delays and impulses, International J. of Computational and Math. Sciences, Vol. 5, № 4, (2011), 229-231. 
[14] Mohamad S., Gopalsamy K., Akça H., Exponential stability of artificial neural networks with distributed delays and large impulses, Nonlinear Analysis: Real World Applications, Vol. 9, № 3, (2008), 872-888.

[15] Özbekler A., Zafer A., Picone type formula for linear non-selfadjoint impulsive differential equations with discontinuous, Electronic J. of Qualitative Theory of Differential Equations, Vol. 35, (2010), 1-12.

[16] Shang Y., The limit behavior of a stochastic logistic model with individual time-dependent rates, Journal of Mathematics, Vol. 2013, (2013), 502635.

[17] Stamov G., Almost periodic solutions of impulsive differential equations, Springer, Heidelberg, New York, Dordrecht, London, (2012).

[18] Stamova I., Stamov G., Lyapunov-Razumikhin method for asymptotic stability of sets for impulsive functional differential equations, Electronic J. of Differential Equations, Vol. 48, (2008), 1-10.

[19] Tariboon J., Ntouyas S., Thaiprayoon C., Asymptotic behavior of solutions of mixed type impulsive neutral differential equations, Advances in Difference Equations, Vol. 2014:327, (2014).
[20] Wang F., Pang G., Shen L., Qualitative analysis and applications of a kind of state-dependent impulsive differential equations, J. of Computational and Applied Math., Vol. 216, № 1, (2008), 279-296.

[21] Wang H., Feng E., Xiu Z., Optimality condition of the nonlinear impulsive system in fed-bath fermentation, Nonlinear Analysis: Theory, Methods \& Applications, Vol. 68, № 1, (2008), 12-23.

[22] Wang W., Shen J., Luo Z., Partial survival and extinction in two competing species with impulses, Nonlinear Analysis: Real World Applications, Vol. 10, № 3, (2009), 1243-1254.

[23] Wang X., Song Q., Song X., Analysis of a stage structured predator-prey Gompertz model with disturbing pulse and delay, Applied Math. Modeling, Vol. 33, № 11, (2009), 4231-4240.

[24] Wu S.-J., Meng X., Boundedness of nonlinear differential systems with impulsive effect on random moments, Acta Mathematicae Applicatae Sinica, Vol. 20, № 1, (2004), 147-154.

[25] Xia Y., Positive periodic solutions for a neutral impulsive delayed Lotka-Volterra competition system with the effect of toxic substance, Nonlinear Analysis: Real World Applications, Vol. 8, № 1, (2007), 204-221. 